\title{
Fermi Surface Manipulation by External Magnetic Field Demonstrated for a Prototypical Ferromagnet
}

E. Młyńczak, ${ }^{1,2,{ }^{*}}$ M. Eschbach, ${ }^{1}$ S. Borek,${ }^{3}$ J. Minár, ${ }^{3,4}$ J. Braun, ${ }^{3}$ I. Aguilera, ${ }^{1}$ G. Bihlmayer, ${ }^{1}$ S. Döring, ${ }^{1}$ M. Gehlmann, ${ }^{1}$ P. Gospodarič, ${ }^{1}$ S. Suga ${ }^{1,5}$ L. Plucinski, ${ }^{1}$ S. Blügel,${ }^{1}$ H. Ebert, ${ }^{3}$ and C. M. Schneider ${ }^{1}$

${ }^{1}$ Peter Grünberg Institut PGI, Forschungszentrum Jülich and JARA- Fundamentals of Future Information Technologies, 52425 Jülich, Germany

${ }^{2}$ Faculty of Physics and Applied Computer Science, AGH University of Science and Technology, al. Mickiewicza 30, 30-059 Kraków, Poland

${ }^{3}$ Department Chemie, Ludwig-Maximilians-Universität München, Butenandtstrasse 5-13, 81377 München, Germany

${ }^{4}$ New Technologies-Research Centre, University of West Bohemia, Univerzitni 8, 30614 Pilsen, Czech Republic

${ }^{5}$ Institute of Scientific and Industrial Research, Osaka University, Ibaraki, Osaka 567-0047, Japan (Received 1 May 2016; revised manuscript received 12 October 2016; published 9 December 2016)

We consider the details of the near-surface electronic band structure of a prototypical ferromagnet, $\mathrm{Fe}(001)$. Using high-resolution angle-resolved photoemission spectroscopy, we demonstrate openings of the spin-orbit-induced electronic band gaps near the Fermi level. The band gaps, and thus the Fermi surface, can be manipulated by changing the remanent magnetization direction. The effect is of the order of $\Delta E=100 \mathrm{meV}$ and $\Delta k=0.1 \AA^{-1}$. We show that the observed dispersions are dominated by the bulk band structure. First-principles calculations and one-step photoemission calculations suggest that the effect is related to changes in the electronic ground state and not caused by the photoemission process itself. The symmetry of the effect indicates that the observed electronic bulk states are influenced by the presence of the surface, which might be understood as related to a Rashba-type effect. By pinpointing the regions in the electronic band structure where the switchable band gaps occur, we demonstrate the significance of spinorbit interaction even for elements as light as $3 d$ ferromagnets. These results set a new paradigm for the investigations of spin-orbit effects in the spintronic materials. The same methodology could be used in the bottom-up design of the devices based on the switching of spin-orbit gaps such as electric-field control of magnetic anisotropy or tunneling anisotropic magnetoresistance.

DOI: 10.1103/PhysRevX.6.041048

Subject Areas: Condensed Matter Physics, Magnetism

\section{INTRODUCTION}

The electronic band structure near the Fermi level determines numerous vital properties of metallic materials, being responsible for their thermal, magnetic, and electronic transport behavior. In the case of metallic ferromagnets, the electronic band structure is split into the minority and majority spin states, as a result of the exchange interaction which rules the relative arrangement of the spins. What binds the spin direction to the orbital degrees of freedom is a relatively weak coupling, the spin-orbit interaction (SOI). The influence of the SOI on the electronic band structure of a ferromagnet is very subtle. It causes the mixing of the spin character and a magnetization-dependent opening of minute energy gaps (about

\footnotetext{
*e.mlynczak@fz-juelich.de
}

Published by the American Physical Society under the terms of the Creative Commons Attribution 3.0 License. Further distribution of this work must maintain attribution to the author(s) and the published article's title, journal citation, and DOI.
$100 \mathrm{meV}$ ) but only at specific points in the reciprocal space. The consequences of these delicate modifications are, however, tremendous. The spin-orbit gaps (SOG) located at the Fermi level, referred to as magnetic monopoles in momentum space [1], are responsible for the magnitude of the intrinsic anomalous Hall effect [1,2], anisotropic magnetoresistance [3], and occurrence of the magnetocrystalline anisotropy (MCA) [4]. Recently, an exciting functionality of the electric-field control of the MCA in itinerant thin-film ferromagnets has been demonstrated $[5,6]$, which paves the way to the novel memory devices with ultralow power consumption. Nakamura et al. [7] explained this effect by showing how an external electric field causes openings of the energy gaps near the Fermi level for the bands that are degenerate when no field is applied.

The SOI-related modifications of the electronic structure near the Fermi level are also the basis for a new generation of magnetic tunnel junctions. In 2002, it was experimentally shown by scanning tunneling microscopy that tunneling current depends on the direction of sample magnetization, even when measured with a 
nonmagnetic tip [8]. This effect, which occurs because of the opening or closing of the SOG for different magnetization directions of the electrode, was later termed tunneling anisotropic magnetoresistance (TAMR) [9]. It was demonstrated, first for the ferromagnetic (FM) semiconductor [9] and later for the conventional ferromagnet ( $\mathrm{Fe}$ ) [10], that thanks to TAMR it is possible to realize a spinvalve function using only one FM electrode. This effect seems to be especially promising for the antiferromagnetic spintronics, where TAMR values of $160 \%$ at low temperatures have been reported [11]. A similar phenomenon, called ballistic anisotropic magnetoresistance (BAMR), was observed in structures where ballistic conductance takes place, such as in nanowires [12]. BAMR occurs because the conductance is directly related to the number of open conducting channels, which might be different along and perpendicular to the nanowire [12].

Moreover, the widely used experimental technique of the x-ray magnetic linear dichroism provides information about the magnetic moment direction because of the dependence of the unoccupied electronic band structure on the magnetization direction [13].

From the point of view of spin dynamics, SOG constitute so-called "spin-flip hot spots," which are crucial for the spin-relaxation processes that govern ultrafast demagnetization [14,15], as well as transmission of the spin currents through the nonmagnetic metals $[16,17]$.

Here, we report on the direct experimental observation of the magnetization-dependent opening of the SOG near the Fermi level. As a subject of this study, we chose Fe(001) thin films grown epitaxially on an $\mathrm{Au}(001)$ single crystal, which can be considered as a prototypical magnetic system. $\mathrm{Fe}$ grown on $\mathrm{Au}(001)$ is characterized by a very low lattice mismatch $(0.6 \%)$, which results in minute strain experienced by the Fe film. Various magnetic and electronic phenomena such as ferromagnetism in the monolayer (ML) regime [18], the existence of quantum well states [19], or the thickness-driven spin-reorientation transition [20] have been studied in this system in the past. We analyzed the electronic structure of the $\mathrm{Fe}(001) / \mathrm{Au}(001)$ system using angle-resolved photoemission spectroscopy (ARPES) for four different remanent in-plane magnetization directions of the FM film, thus exploring the electronic origins of the SOI-related effects. Our experiments revealed distinct changes in the position of electronic bands near the $\bar{X}$ point of the surface Brillouin zone (SBZ) in response to the change of the magnetization direction. To interpret the experimental results in terms of the bulk electronic structure, we performed calculations using Green's function formalism within the $G W$ scheme. For the discussion of the electronic structure of the $\mathrm{Fe}(001)$ surface, we employed slab calculations based on the generalized gradient approximation (GGA). A theoretical treatment of the entire photoemission process was addressed using state-of-theart, one-step model photoemission calculations.

\section{EXPERIMENT AND CALCULATION}

\section{A. Experiment}

The 100-ML Fe films $(1 \mathrm{ML}=1.43 \AA)$ investigated in this study were deposited by molecular beam epitaxy onto the $\mathrm{Au}(001)$ single-crystal surface. The $\mathrm{Au}(001)$ template was prepared by repeated cycles of Ar sputtering and subsequent annealing at $500{ }^{\circ} \mathrm{C}$ for $10 \mathrm{~min}$, until the wellknown surface reconstruction [21] was clearly visible using low-energy electron diffraction (LEED). Fe deposition was performed at a temperature of $\mathrm{T}=50 \mathrm{~K}$. After deposition, the Fe films were briefly heated up to $300{ }^{\circ} \mathrm{C}$. To address the problem of the possible diffusion of the Au atoms towards the $\mathrm{Fe}$ surface in our system, we performed a supplementary study combining ultraviolet photoemission spectroscopy (UPS) using a He I emission line ( $h \nu=$ $21.2 \mathrm{eV}$ ) and x-ray photoelectron spectroscopy (XPS) (Appendix A). The results obtained show that the Fe surface was free from the Au adatoms, within the sensitivity limit of our spectroscopic determination, which we can safely assume to be of the order of $0.1 \mathrm{ML} \mathrm{Au}$.

Before each ARPES measurement, the samples were remanently magnetized. The external magnetic field was applied to the thin-film sample by an oriented permanent magnet (about $20 \mathrm{mT}$ ), which was brought close to the sample surface (as close as $2-3 \mathrm{~cm}$ ) in an air well set on a linear feedthrough. The procedure of the magnetization was similar to the one used in Ref. [22]. Stray fields caused by the remanent magnetization of the Fe(001) film do not distort the trajectories of emitted photoelectrons due to the very small Fe volume. For the Fe thickness range used in this study, the easy magnetization directions of $\mathrm{Fe}(001)$ are in plane [20], and because of the low coercivity of Fe film $\left(\mathrm{H}_{\mathrm{C}}=1-2 \mathrm{mT}\right)$, a remanent magnetization is easily realized. ARPES measurements were performed for samples magnetized along [100], [100], [010], and [010], which will be referred to as UP, DOWN, RIGHT, and LEFT, respectively. Figure 1 shows a sketch of the experimental geometry. The ARPES experiments were performed with a laboratory-based hemispherical electron energy analyzer (MB Scientific A-1) using unpolarized nonmonochromatized neon emission from a SPECS UVS-300 discharge lamp with a focusing capillary. During ARPES measurements, the $\mathrm{Au}(001)$ substrate was kept at a temperature of $\mathrm{T}=50 \mathrm{~K}$, which prevented condensation of the neon atoms on the sample surface. All the ARPES spectra discussed in this study were collected in the energy region close to the Fermi level (within a binding energy range of $200 \mathrm{meV}$ ), utilizing the higher-energy neon emission line of $h \nu=16.85 \mathrm{eV}$. The analyzer was set to an energy resolution of $10 \mathrm{meV}$ for all the presented spectra. When electrons are detected along the sample normal $\left(\Theta=0^{\circ}\right)$, the light impinges under a grazing angle of $\phi=15^{\circ}$ with respect to the sample surface, which lies in the $x y$ plane of the laboratory reference frame. To measure the Fermi 


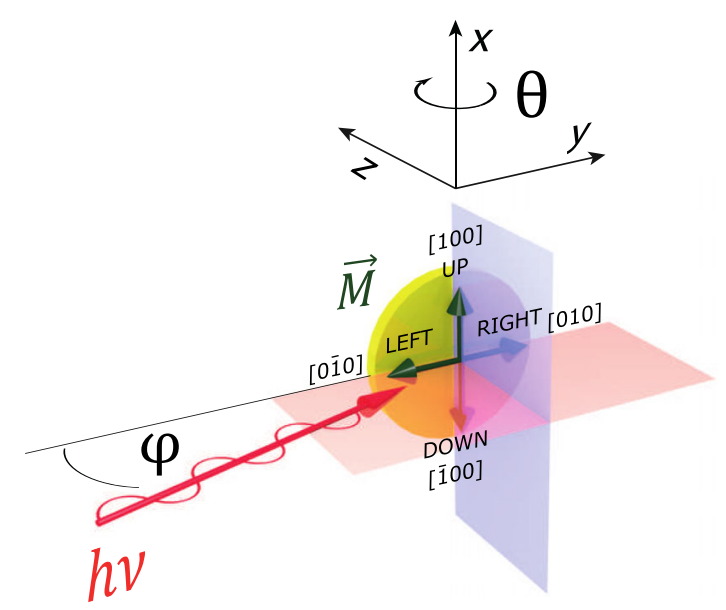

FIG. 1. Schematic representation of the experimental ARPES geometry. Light incidence and electron emission planes are indicated by a red (horizontal) and a blue (vertical) plane, respectively. Black arrows represent magnetization directions referred to as UP, DOWN, LEFT, and RIGHT.

surface, the sample was rotated around the $x$ axis, as the change in angle $\Theta$ corresponds to a change in $k_{\| y}$, according to $k_{\| y}=\sqrt{\left(2 m / \hbar^{2}\right) E_{\text {kin }}} \sin \Theta$, where $E_{\text {kin }}$ represents the kinetic energy of photoemitted electrons. Therefore, the light incidence angle $\left(\phi=15^{\circ}+\Theta\right)$ changed during scanning of the angle $\Theta$, reaching $53^{\circ}$ at the $\overline{\mathrm{X}}$ point, in the vicinity of which the key measurements were performed. The magnetization-dependent bandstructure features observed in ARPES were perfectly reproducible for multiple remagnetization cycles.

\section{B. Calculation}

In order to obtain the theoretical bulk band structure of body-centered cubic (bcc) Fe, many-body calculations in the framework of the $G W$ approximation were carried out within the all-electron full-potential linearized augmentedplane-wave (FLAPW) formalism as implemented in the SPEX code [23]. For simulating the surfaces, we employed density functional theory (DFT) in the GGA in the form of Perdew et al. [24]. We used 27 layers of $\mathrm{Fe}(001)$ and relaxed the outermost four layers using the film version of the FLEUR code [25]. Spin-orbit coupling was included selfconsistently in the calculations. For the assessment of the Fermi surfaces, a $39 \times 39 k$-point grid was used.

To theoretically analyze the photoemission process, onestep model photoemission calculations were performed. These calculations can be subdivided into two main parts. In the first part, one has to determine the electronic structure of the system. Therefore, we set up a semi-infinite system that simulates the Fe(001) surface. For the calculation of the ground-state properties, we used a fully relativistic multiple scattering method in the framework of density functional theory (Korringa-Kohn-Rostoker, KKR) [26]. In the second part, the fully relativistic one-step model of photoemission was applied. The approach goes back to the developments worked out by Pendry and coworkers [27-29]. At this stage, we calculated the elastic part of the photocurrent and neglected the interaction of the outgoing photoelectron with the rest of the electronic system (sudden approximation). For the calculation of the matrix elements that define the transition probability of the photoelectron, one has to consider the initial- and final-state wave functions. The final state has been constructed using the theory of spin-polarized low-energy electron diffraction (SPLEED). In this framework, the final state is represented by a so-called time-reversed SPLEED state [30,31]. Using a parametrized and energy-dependent inner potential, we addressed the many-body interactions. We therefore corrected the elastic part of the photocurrent for inelastic interactions phenomenologically. For the escape of the photoelectron into vacuum, one has to consider a surface barrier for which we used the parametrization of Rundgren and Malmström [32]. The barrier can be treated straightforwardly as an additional surface layer, and it accounts for surface contributions to the photocurrent. Furthermore, we can investigate the various possible transitions separately by the suppression of predetermined initial states, which offers the possibility to analyze the main contribution to the transitions according to the dipole selection rules. Because the light source used in the experiment is unpolarized, we consider a 50\% mixture of the results obtained for the calculations for $p$ - and $s$-polarized light.

\section{RESULTS AND DISCUSSION}

\section{A. Spin-orbit coupling signatures in the bulk electronic band structure of bcc Fe}

Figure 2(a) shows the sketch of the bcc bulk Brillouin zone (BBZ) and the (001) surface Brillouin zone (SBZ), which we will refer to frequently when discussing electronic band dispersions. An arrow indicates the magnetization direction. Note that the $\overline{\mathrm{X}}$ point is the projection along $k_{\|}=0.5|\Gamma-\mathrm{H}|$. Band dispersions found for the $\Gamma-\mathrm{H}$ $(\Delta)$ line of the bulk Brillouin zone using the $G W$ method are presented in Fig. 2(b). Two directions are distinguished: parallel and perpendicular to the magnetization $(\overrightarrow{\mathrm{M}})$ (plotted for positive and negative $k$ values, respectively). The color code refers to the spin character of each band; blue (red) marks minority (majority) spin states. Because of the introduction of spin-orbit interaction, electronic states of opposite spin become significantly mixed in the vicinity of the points where the bands would cross if no spin-orbit interaction was present. As a result, not only purely minority and majority states exist but also states with mixed spin character. The bands are marked according to the single group representation, which is frequently used in the literature, especially in the discussions of the tunneling effect in the $\mathrm{Fe} / \mathrm{MgO} / \mathrm{Fe}$ magnetic tunnel junctions [33]. The small Greek letters $(\alpha, \beta, \gamma, \delta)$ correspond to the 


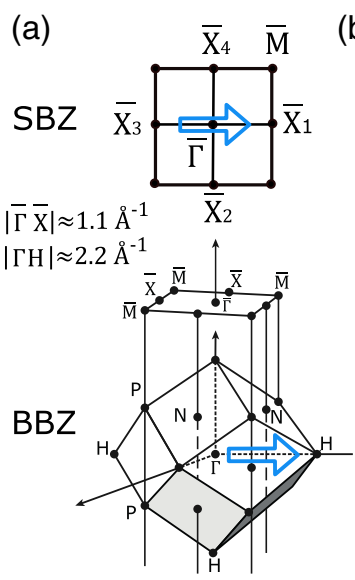

(b)

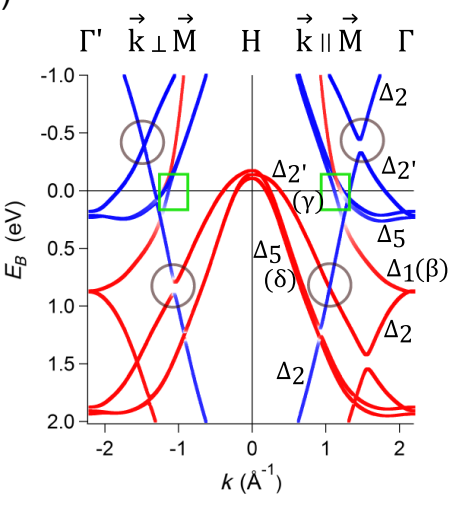

(c)

no SOI

$\overrightarrow{\mathrm{k}} \| \overrightarrow{\mathrm{M}}$

$\overrightarrow{\mathrm{k}} \perp \overrightarrow{\mathrm{M}}$
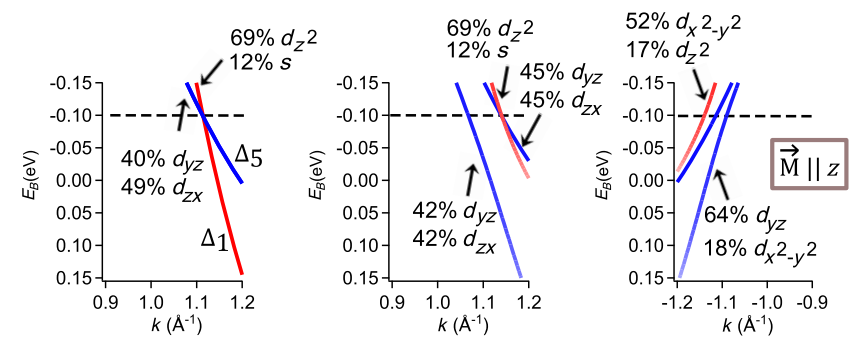

FIG. 2. (a) Bulk and (001) surface Brillouin zones of bcc Fe. High symmetry points $\bar{X}_{1}$ to $\bar{X}_{4}$ are defined with respect to the magnetization direction depicted by an arrow. (b) Relativistic bulk band structure of bcc Fe, calculated along the $\mathrm{H}-\Gamma$ (positive $k$ ) and $\mathrm{H}-\Gamma^{\prime}$ (negative $k$ ) using the $G W$ method. Circles mark band crossings or SOG mentioned in the text. Green rectangles mark regions enlarged in (c). (c) Place of interest near $k=0.5|\Gamma-\mathrm{H}|$, close to the Fermi level [shown by green rectangles in (b)], where three bands cross in a magnetization-dependent manner. The left panel shows the nonrelativistic situation (no SOI), in the center panel the $k$ vectors are parallel to $\overrightarrow{\mathrm{M}}$, and the right plot shows $k$ vectors perpendicular to $\overrightarrow{\mathrm{M}}$. Blue (red) lines indicate predominantly minority (majority) spin. The magnetization $(\vec{M})$ is parallel to the $z$ axis. The horizontal dashed line marks the position of the experimental Fermi level.

labeling of the Fermi sheets used later in this article to identify the experimentally observed spectral signatures.

We observe considerable differences between the band structures for the direction along the magnetization $\vec{M}$ and perpendicular to the magnetization [Fig. 2(b)]. For example, the crossing of the minority spin $\Delta_{2}$ and $\Delta_{2}{ }^{\prime}$ bands right above the Fermi level develops a gap for the direction parallel to the magnetization [marked with circles in Fig. 2(b)]. Such an avoided crossing is also observed for the minority $\Delta_{2}$ and majority $\Delta_{2}^{\prime}$ bands that cross near $E_{B}=1 \mathrm{eV}$ for the $k$ vectors along the magnetization but show a gap opening for the perpendicular direction. As expected, crossing points for which hybridization of the bands occurs and the spin-orbit energy gaps open depend not only on the spatial part of the respective wave functions but also on the direction of the spin within each band. The size of the spin-orbit gaps reaches $\Delta E_{\mathrm{SO}} \sim 120 \mathrm{meV}$.

The electronic band structure near the Fermi level is of particular interest because of its influence on magnetocrystalline anisotropy and electronic transport. Therefore, we identified the places of interest in the bulk Brillouin zone along the $\Delta$ line that showed magnetizationdependent openings of spin-orbit gaps. These regions are marked by green rectangles in Fig. 2(b) and shown in detail in Fig. 2(c). For the determination of the orbital symmetries, we define magnetization $\vec{M}$ as being parallel to the $z$ axis. The predominant orbital characters of each band, which govern the occurrence of the SOI-induced hybridization gaps, are listed as follows: On the left-hand side of Fig. 2(c), we plot the dispersion of the $\Delta_{1}$ band (predominantly $d_{z^{2}}$ orbital symmetry) and the doubly degenerated $\Delta_{5}$ band ( $d_{y z}+d_{z x}$ orbital symmetry) calculated without spin-orbit interaction. In the relativistic case, i.e., when the spin-orbit interaction is included in the calculation [Fig. 2(c), middle and right panels], the $\Delta_{5}$ band splits and hybridizes with the $\Delta_{1}$ band, as $\left\langle\Psi\left(d_{z^{2}} \uparrow\right)\left|H_{\text {SO }}\right| \Psi\left(d_{y z(z x)} \downarrow\right)\right\rangle \neq 0$. For the $k$ vectors parallel to $\overrightarrow{\mathrm{M}}$, the bands of $d_{z^{2}}$ and $d_{y z}\left(d_{z x}\right)$ symmetry can still cross [Fig. 2(c), middle panel]. However, for the $k$ vectors perpendicular to $\overrightarrow{\mathrm{M}}$, all three bands avoid crossing each other [Fig. 2(c), right panel]. Therefore, we expect an experimentally observable difference between these two directions in $k$ space in the vicinity of the $k_{\|}=1.0-1.2 \AA^{-1}$, i.e., near the $\overline{\mathrm{X}}$ point of the SBZ.

\section{B. Band structure of $\mathrm{Fe} / \mathrm{Au}(001)$ : Experiment and theory}

ARPES spectra were recorded for a series of polar angles $\Theta$ spanning the range of the SBZ that contain the $\bar{\Gamma}\left(k_{y}=0 \AA^{-1}\right)$ and $\bar{X}$ points $\left(k_{y} \sim 1.1 \AA^{-1}\right)$ [see Fig. 1 and Fig. 2(a) for the sketch of the SBZ]. The obtained data set measured for the sample magnetized RIGHT is shown in Fig. 3 as a band map along the $\bar{\Gamma}-\bar{X}$ line (a) and the corresponding constant energy cut at the Fermi level [ $E_{F}\left(k_{x}, k_{y}\right)$, later referred to as the Fermi surface] (b). The arrows mark the magnetization direction. The calculated dispersions presented in Fig. 3(a) show $G W$ bulk electronic structure of bcc $\mathrm{Fe}$, along the magnetization direction, for $k_{\perp} \sim 2.2 \AA^{-1}$, i.e., near the $\mathrm{H}$ point (the $k_{\perp}$ value was determined based on the free-electron final-state model; see Appendix B for details). The dispersions are therefore the same as shown in Fig. 2(b) (positive k values) and Fig. 2(c) (middle panel). The binding energy of $E_{B}=-0.1 \mathrm{eV}$ in the theoretical result was aligned with the experimental Fermi level. The theoretical cut through the Fermi surface [Figs. 3(c) and 3(f)] was constructed from the calculated dispersions, taking into account the energy range defined by the Gaussian function 


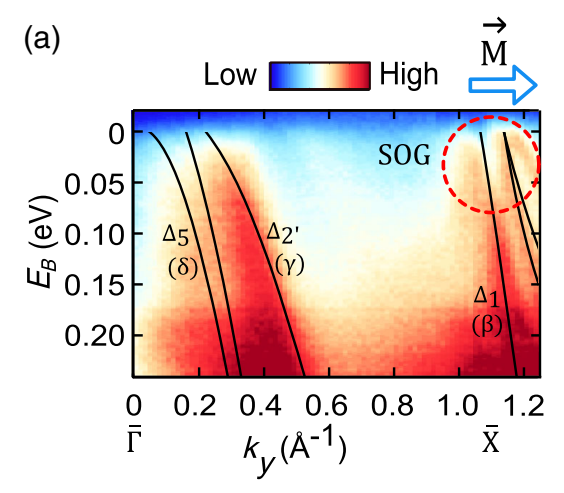

(d)
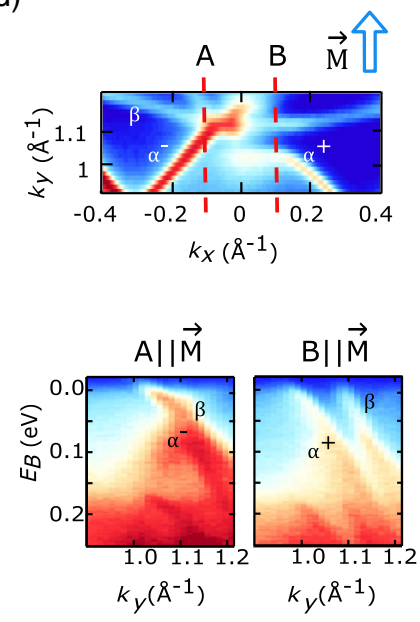

(b)

(e)

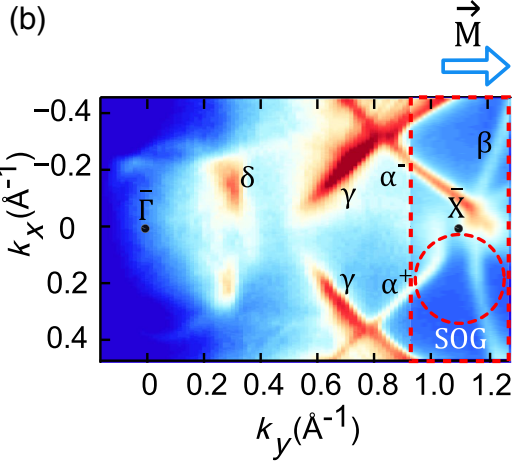

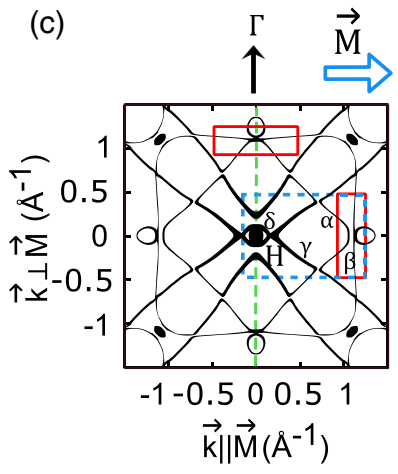

(f)
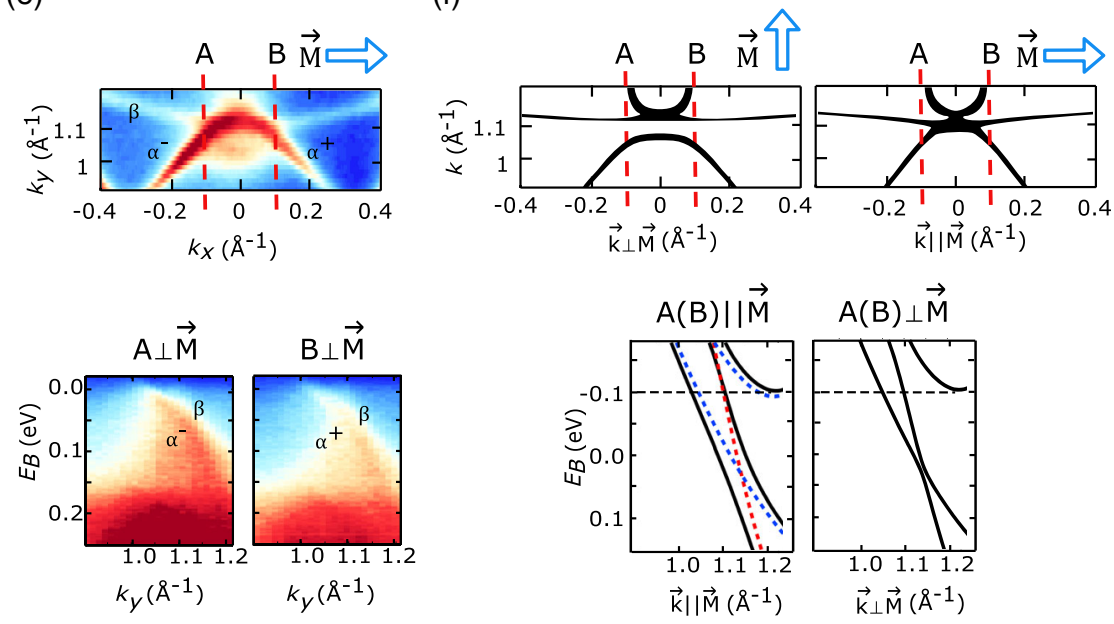

FIG. 3. (a) ARPES spectra obtained for the binding energies close to the Fermi level, along the $\bar{\Gamma}-\overline{\mathrm{X}}$ direction for the magnetization RIGHT. Black lines are superimposed results of the $G W$ calculations of the bulk electronic structure of Fe for $k_{\perp} \sim 2.2 \AA^{-1}$, which, according to the free-electron final-state model, corresponds to the excitations induced by $h \nu=16.8 \mathrm{eV}$. Calculated dispersions are the same as the ones depicted in Fig. 2(b) (H- $\Gamma$ direction). (b) Fermi surface in the vicinity of the $\bar{\Gamma}-\bar{X}$ direction measured for the magnetization RIGHT. The red rectangle on the right marks the region where detailed spectra for other magnetization directions were obtained [(d), (e) and Fig. 5(a)]. Dashed circles in (a) and (b) indicate the observed SOG. (c) $G W$ calculations of the bulk electronic structure of Fe for $k_{\perp} \sim 2.2 \AA^{-1}$. The blue dashed rectangle marks the $k$-space region shown in (b); red rectangles mark the $k$ space where the detailed spectra for all the magnetization directions were obtained [(d), (e), and Fig. 5(a)]. (d) Zoom of the Fermi surface measured in the region of the red rectangle marked in (b) (90 rotated) together with the dispersions for $\left|k_{x}\right|=0.1 \AA^{-1}$ (along two dashed red lines) marked as $A$ and $B$. (e) The same as (d), measured for the magnetization DOWN. (f) Zooms within the area of the two red rectangles marked in (c) together with the theoretical dispersions along the red dashed lines marked as $A$ and $B$. Dashed lines in the bottom right panel are the bands calculated without the introduction of SOI: Blue (red) indicates minority (majority) spin. Arrows indicate magnetization direction.

of $\sigma=8 \mathrm{meV}$. This cut presents a plane within which the magnetization vector lies (the magnetization direction is indicated by an arrow), which allows the difference to be captured between the $k$-space directions, along the magnetization (horizontal) and perpendicular to the magnetization (vertical). The regions of interest, where the magnetization-dependent opening of the SOG is expected, are indicated by red rectangles [these regions are enlarged in Fig. 3(f)]. The blue dashed rectangle marks the $k$-space region that corresponds to the experimental result depicted in (b).

The observed electronic bands [Fig. 3(a)] are sharpest near the Fermi level but become diffuse for larger binding energies of the order of $100 \mathrm{meV}$, possibly due to the influence of the electron correlation effects in the system [34]. For binding energies larger than $E_{B}=180 \mathrm{meV}$, a spectral intensity from the second neon emission line $(h \nu=16.67 \mathrm{eV})$ is visible. Figures 3(a)-3(c) show that the experimentally observed electronic bands were found to match the result of the bulk $G W$ calculations reasonably well. The Fermi surface sheets and the corresponding bands are marked with small Greek letters. Comparing the experimental Fermi surface [Fig. 3(b)] to the theoretical prediction [Fig. 3(c)], we can identify characteristic shapes of the $\alpha$ and $\gamma$ sheets [although the $\alpha$ sheet cannot be traced along the $\bar{\Gamma}-\bar{X}$ line in Fig. 3(a), it is very clearly observed for nonzero $k_{x}$ ]. The experimental $\alpha$ sheet is apparently nonsymmetric with respect to the $k_{x}=0 \AA^{-1}$; compare $\alpha$ 
(a)

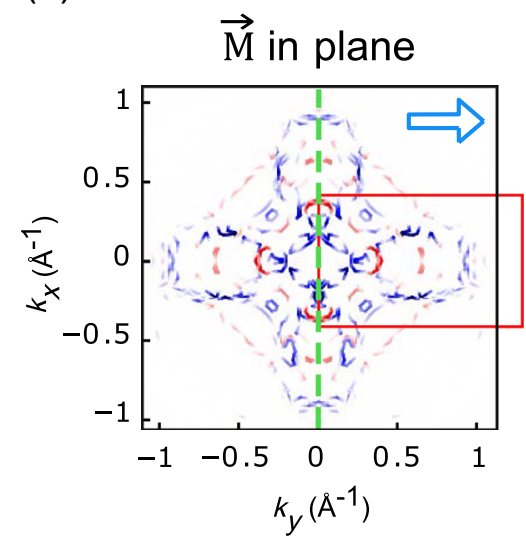

(b)

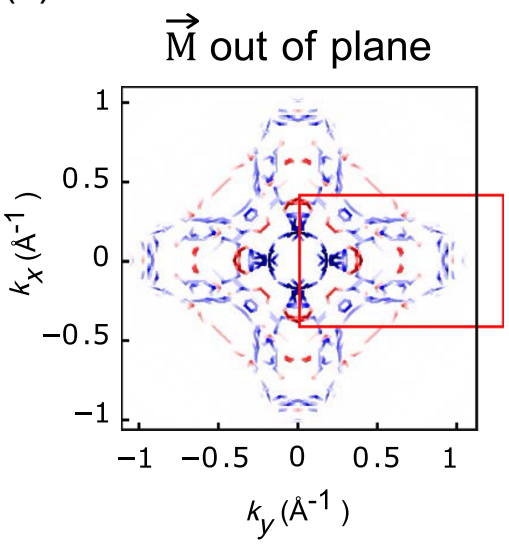

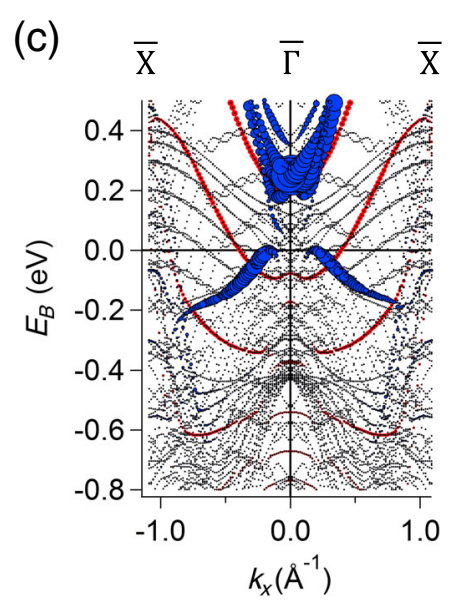

FIG. 4. Results of the relativistic GGA slab calculations that reveal the surface electronic structure of Fe(001). (a) Magnetization in plane. The dashed vertical line marks the mirror plane. (b) Magnetization out of plane. The red rectangles show the fraction of the $k$ space measured in the experiment [Fig. 3(b)]. (c) Dispersions along the $\bar{\Gamma}-\bar{X}$ direction. The size of the symbol corresponds to the localization above the surface layer. Magnetization lies in plane, along the $k_{y}$ axis. Blue (red) symbols correspond to the predominantly minority (majority) states.

sheets $\alpha^{+}$and $\alpha^{-}$(branches found for positive and negative $k_{x}$ values, respectively). The observed asymmetry will be analyzed in detail in the discussion of Figs. 4 and 5. The experimental $\gamma$ sheet reaches the $k_{x}=0 \AA^{-1}$ line at $k_{y} \sim 0.5 \AA^{-1}$ (with vanishing intensity for $k_{x}=0$ ), which is further from the $\bar{\Gamma}$ point than theoretically predicted. Another prominent feature that we find close to the $\overline{\mathrm{X}}$ point is the $\beta$ sheet, which, in three dimensions $\left(k_{x}, k_{y}, k_{z}\right)$, exhibits a cubelike shape. In the ARPES spectra, we also observe a significant spectral contribution near $k_{x(y)} \sim$ $0.2 \AA^{-1}$, which can be attributed to the Fermi sheet $\delta$ even though the spin-orbit splitting of this state cannot be resolved. In Fig. 5, we show that the experimental spectral intensity in this region of $k$ space is well reproduced by one-step model photoemission calculations. The three bands visible in the experiment close to the $k_{y} \sim 1.1 \AA^{-1}$ [Fig. 3(a)] correspond to the $\Delta_{1}$ band and the spin-orbit split $\Delta_{5}$ band [compare with Fig. 2(c), left and middle panels]. Therefore, our experiment directly shows the spinorbit gap expected to occur for $k$ vectors along the magnetization direction. We observe the SOG both as a function of binding energy [Fig. 3(a)] and momentum [Fig. 3(b)] (marked by a red dashed circle). Based on the bulk $G W$ calculations [Figs. 2(b), 2(c), and 3(c)], we expect the closing of the SOG near the $\overline{\mathrm{X}}$ point for $k$ vectors perpendicular to the magnetization direction.

The $k$-space region measured in the experiment for the magnetization RIGHT near the $\overline{\mathrm{X}}$ point is reproduced in Fig. 3(d). The Fermi surface within the same region of the $k$ space measured after magnetizing the sample in the direction DOWN is shown in Fig. 3(e). The arrows in Figs. 3(d) and 3(e) indicate the remanent magnetization direction of the sample during these two measurements. In addition, band dispersions for wave vectors with $\left|k_{x}\right|=$ $0.1 \AA^{-1}$ are shown in the lower left and right panels, $A$ and $B$ (cuts along the lines $A$ and $B$ marked in the upper panels).

Clearly, the experimental intensity distribution depends on the magnetization direction. For the magnetization RIGHT [Fig. 3(d)], we experimentally observe two bands located closer to each other (cut $A$ ) or clearly separated from each other (cut $B$ ). For magnetization DOWN [Fig. 3(e)] along both $A$ and $B$ cuts, bands located close to each other are observed. These experimental dispersions can be compared to the dispersions predicted by bulk $G W$ calculations. Figure 3(f) presents the zooms within the areas of interest [marked by red rectangles in Fig. 3(c)] and, additionally, dispersions along the lines marked as $A$ and $B$ [to be compared with Figs. 3(d) and 3(e)]. In both cases $(\overrightarrow{\mathrm{k}} \| \overrightarrow{\mathrm{M}}$ and $\overrightarrow{\mathrm{k}} \perp \overrightarrow{\mathrm{M}})$, dispersions along $A$ and $B$ are identical. The two bands show an avoided crossing for both $\overrightarrow{\mathrm{k}} \| \overrightarrow{\mathrm{M}}$ and $\overrightarrow{\mathrm{k}} \perp \overrightarrow{\mathrm{M}}$. This can be seen by comparison with the dispersions calculated without the spin-orbit interaction (shown in the $A(B) \| \overrightarrow{\mathrm{M}}$ panel as blue and red dashed lines that correspond to the minority and majority spin, respectively). The sizes of the resulting gaps are, however, very different for both directions: larger for $\vec{k} \| \vec{M}$ and smaller for $\vec{k} \perp \vec{M}$.

By comparing Figs. 3(d)-3(f), we deduce that the experimentally observed difference in the dispersions and positions of the Fermi sheets between the magnetizations RIGHT and DOWN clearly resemble the difference expected in the bulk electronic structure between the directions $\overrightarrow{\mathrm{k}} \| \overrightarrow{\mathrm{M}}$ and $\vec{k} \perp \vec{M}$. [Fig. 3(f)]. Therefore, we have shown that the experimentally observed effect is of the same character and within the same order of magnitude as expected for the SOI-related shifts of the bulk electronic bands linked to the 


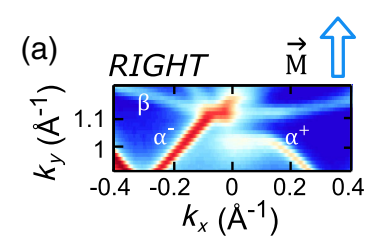

(e)

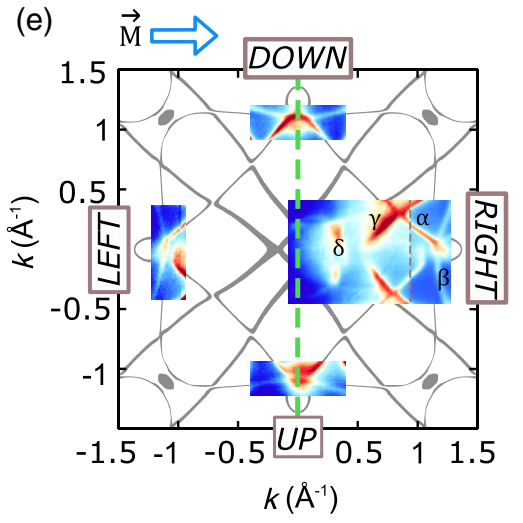

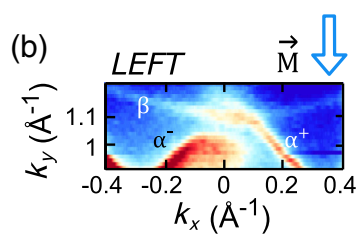

(f)

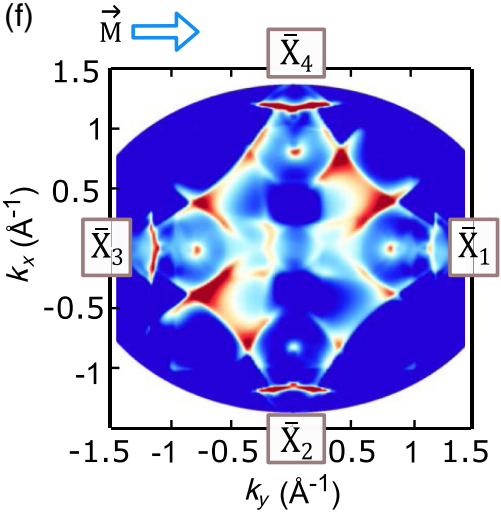

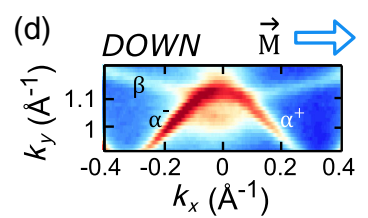

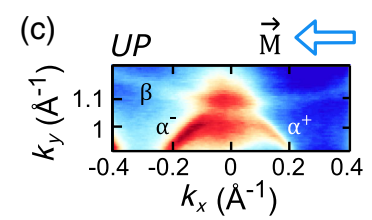

(k)

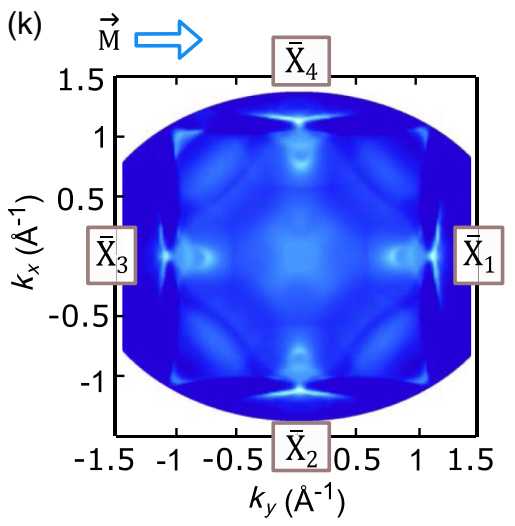

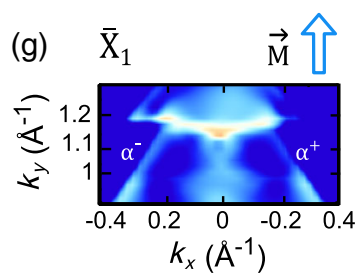
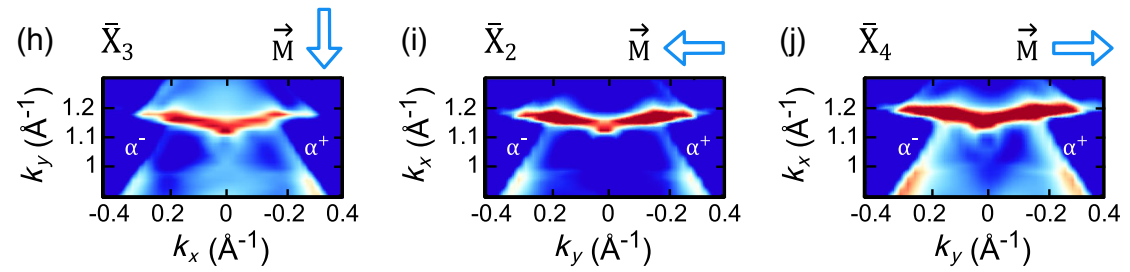

FIG. 5. (a)-(d) Electronic structure of $\mathrm{Fe}(001)$ close to the $\overline{\mathrm{X}}$ point of the SBZ for four different in-plane easy magnetization directions measured at $h \nu=16.8 \mathrm{eV}$. (e) Fermi surfaces derived from (a)-(d) arranged with respect to the fixed magnetization direction (arrow); this approximation is applicable when the role of the light incidence direction is neglected. The gray line depicts the electronic band structure of the bulk $k_{\perp}=2.2 \AA^{-1}$ calculated using the $G W$ method. The dashed vertical line marks the mirror plane. (f) Result of the one-step model photoemission calculation that shows spectral intensities within the entire SBZ. The arrow marks the magnetization direction. (g)-(j) Results of the one-step model photoemission calculation shown for the fractions of the $k$ space that correspond to experimental Fermi surfaces presented in (a)-(d). (k) Result of the one-step model photoemission calculations performed without the $d-f$ transitions. All the one-step model photoemission calculation results shown are obtained with intentionally quenched surface contribution and the light incidence direction along the magnetization.

change of the remanent magnetization direction. However, the exact shape of the experimental Fermi sheet found for $\overrightarrow{\mathrm{k}} \| \overrightarrow{\mathrm{M}}$ (magnetization RIGHT) [Figs. 3(b) and 3(d)], i.e., lack of symmetry with respect to the $\bar{\Gamma}-\overline{\mathrm{X}}$ line, cannot be explained by the bulk electronic structure.

In order to shed more light on this issue, we need to address the role that the surface plays in our photoemission experiment. Figure 4 shows the result of the slab calculations, which reveal the surface electronic structure of $\mathrm{Fe}(001)$ for the magnetization lying in plane [Fig. 4(a)] and out of plane [Fig. 4(b)]. Figure 4(c) presents the band dispersions calculated along $\bar{\Gamma}-\bar{X}\left[k_{x}\right.$ direction, along dashed vertical line in Fig. 4(a)] for the in-plane magnetization lying along the $k_{y}$ axis. Blue (red) symbols correspond to the predominantly minority (majority) states.

The calculations reveal a surface state (SS) of predominantly minority spin character that forms a four-petal-like shape at the Fermi surface. This characteristic state appears in earlier first-principles calculations of the $\mathrm{Fe}(001)$ surface
[35-37]. Our experiment does not show any indications of the surface state, along normal or off-normal emission. Experimentally, diverse electronic states interpreted as surface related were observed for $\mathrm{Fe}(001)$ in the past [35,37-43]. Even restricting the discussion only to the states along sample normal already shows the complexity of the problem: The SS was reported to be occupied $\left(E_{B}=0.3 \mathrm{eV}\right)$ of majority character $[40,43]$, but indications of the minority state at the same binding energy were also reported [43]. Some reports show the surface state along normal to be unoccupied $[35,41]$ of the minority character [41]. Some other works present proof of the absence of the surface state along normal emission, both below the Fermi level [37] and above the Fermi level [42]. It seems that the possible appearance of the surface-related features in the electronic band structure of $\mathrm{Fe}(001)$ critically depends on the Fe surface preparation conditions.

Even though the surface states predicted by the calculation are not revealed in our experiment, the results 
presented in Figs. 4(a) and 4(b) reflect the influence of the magnetization direction on the symmetry of the electronic states when the translational symmetry of the system is broken by the presence of the surface. In the ferromagnetic state, the symmetry of the bulk electronic structure is lower than the full octahedral symmetry of the atomic structure of the Fe bulk crystal. Due to the fact that magnetization is an axial vector, only a single mirror plane can be identified for the bulk electronic structure of $\mathrm{Fe}$, i.e., a plane being perpendicular to magnetization. It is marked in Fig. 3(c) with a dashed vertical line. Additionally, in the bulk, a fourfold rotation axis along the magnetization vector exists, leading to the symmetry group $D_{4 h}$ [44]. When we consider a case of the in-plane magnetized $\mathrm{Fe}(001)$ surface, the rotational symmetry of the bulk is lifted, and only the mirror plane perpendicular to the magnetization is retained $\left[C_{s}\right.$ symmetry, vertical dashed line in Fig. 4(a)]. When the $\mathrm{Fe}(001)$ surface is magnetized out of plane, its symmetry is $C_{4}\left(90^{\circ}\right.$ rotation axis) [Fig. 4(b)]. In a situation of symmetry breaking by the surface and in-plane magnetization [Fig. 4(a)], electronic bands observed for two opposite $k$ vectors that are perpendicular to the magnetization direction (namely, $+k_{x}$ and $-k_{x}$ ) are not equivalent. This effect can be seen within the area marked by the red rectangle in Figs. 4(a) and 4(c). The very same type of asymmetry was found in our experiment for the magnetization RIGHT [Figs. 3(b) and 3(d)]. In addition, the symmetry observed for the measurement performed when the sample was magnetized DOWN [Fig. 3(e)] is consistent with the existence of the mirror plane that is perpendicular to the magnetization direction.

In order to confirm that the symmetry of the states measured with ARPES agrees with the symmetry expected for the surface states, we measured ARPES spectra around the same $\bar{X}$ point with respect to the experimental reference frame for the other remanent magnetization directions, i.e., LEFT and UP. The obtained Fermi surfaces are summarized in Figs. 5(a)-5(d). Figures 5(a) and 5(d) present the results of the measurement for the magnetization RIGHT and DOWN, which were already shown in Figs. 3(d) and 3(e). Now we can compare these to the spectral weights observed for other magnetization directions.

We see that the SOG found for the magnetization RIGHT [Fig. 5(a)] is also visible when the magnetization is pointing LEFT. For the magnetization RIGHT, the SOG was observed for the $\alpha^{+}$sheet, while for the magnetization LEFT, it is visible for the $\alpha^{-}$sheet [Fig. 5(b)]. The size of the observed spin-orbit gap is of the order of $0.1 \AA^{-1}$. However, when the magnetization direction points UP or DOWN [Figs. 5(c) and 5(d), respectively], both $\alpha^{+}$and $\alpha^{-}$ are rather symmetric with respect to the $\bar{\Gamma}-\overline{\mathrm{X}}\left(k_{x}=0 \AA^{-1}\right)$ line, and no clear gap is visible. However, the difference observed between these two magnetization directions is the shift of the spectral weight towards higher $k_{y}$ values for the magnetization DOWN, as compared to UP.
The illustration presented in Fig. 5(e) shows how the measured spectra can be arranged with respect to the magnetization direction when the role of the light incidence direction is neglected, which allows the identification of the symmetries characteristic of the observed bands. The superimposed gray solid line in the illustration shows the theoretical (bulk $G W$ ) cut through the Fermi surface of bcc Fe at $k_{\perp} \sim 2.2 \AA^{-1}$. The experimental result reveals only the single mirror plane (vertical dashed line), perpendicular to the magnetization direction, i.e., the symmetry point group $C_{s}$, which is identical to the one found for the electronic structure of the surface [Fig. 4(a)]. Therefore, we confirmed that the experimentally observed electronic bands show the symmetry expected for the surface electronic structure. Next, we verify if this experimental observation is reproduced in the one-step model calculations of the photoemission process.

The result of the one-step model calculations of photoemission induced by light of $h \nu=16.8 \mathrm{eV}$ from Fe(001) in the geometry of our experiment is presented in Fig. 5(f). Figures 5(g)-5(j) show magnified regions of the one-step model calculation results in the fractions of the $k$ space that correspond to the measurements shown in Figs. 5(a)-5(d). The projection of the light incidence direction on the sample surface lies along the magnetization direction [indicated by an arrow in Fig. 5(f)]. In the calculation, the spectral intensity originating from the surface states was intentionally quenched (see Appendix $\mathrm{C}$ for the computational details), according to the good correspondence of our experiment and the bulk electronic structure of bcc Fe. In this way, we can theoretically examine how the bulk states located close to the sample surface are affected by the broken translational symmetry.

One can recognize the close resemblance of the results of the one-step model calculations, the results of the experiment, and the theoretical bulk electronic structure cut at $k_{\perp} \sim 2.2 \AA^{-1}$ [Figs. 5(e) and 5(f)]. Very good agreement concerning the intensity of the photoemission features between experiment and one-step model calculations is revealed, especially for the $\gamma$ Fermi sheet [Figs. 5(e) and 5(f)]. The $k$-space region near the $\overline{\mathrm{X}}$ points is also relatively well reproduced by the calculations. The comparison of Figs. 5(a)-5(d) and 5(g)-5(j) shows that even though the exact shifts of the $\alpha$ sheet observed in experiment are not visible in the calculations, the overall symmetry reflects the experimental one. The $k$-space regions in the vicinity of the points $\bar{X}_{1}$ [Fig. $\left.5(\mathrm{~g})\right]$ and $\bar{X}_{3}$ [Fig. 5(h)] [which correspond to the measurements performed with the magnetization pointing RIGHT, Fig. 5(a), and LEFT, Fig. 5(b), respectively] are not symmetric with respect to the $k_{x}=0$ line. The sheets $\alpha^{+}$ are less (more) intense than $\alpha^{-}$for the point $\overline{\mathrm{X}}_{1}\left(\overline{\mathrm{X}}_{3}\right)$, which resembles the experimentally found shift of the $\alpha$ sheet. Both $\alpha$ branches are less intense near the $\overline{\mathrm{X}}_{2}$ point [Fig. 5(i)] than near the point $\bar{X}_{4}$ [Fig. 5(j)], which corresponds to the 
bigger distance between the $\alpha$ and $\beta$ Fermi sheets observed in the experiment for the magnetization UP [Fig. 5(c)].

In addition, to access the information on the final states involved in the observed transitions, we performed one-step model photoemission calculations neglecting the excitations from $d$ to $f$ states. The result is presented in Fig. 5(k). We see that the remaining spectral intensity is relatively weak, and the spectral features that we could compare to the experimental result vanish. Therefore, we show that the electronic transitions seen in our ARPES experiment are mainly from the initial states of $d$ character to the final states of $f$ character.

Based on the extensive analysis of the experimental results and the theoretical calculations, we can conclude that we experimentally observed the spin-orbit interactionrelated and magnetization-dependent band gaps within the bulk states of bcc Fe. The asymmetry of the bulk electronic bands found in our experiment is consistent with the one expected for a system where both exchange coupling and spin-orbit interaction coexist, when the existence of the sample surface is taken into account.

Even though Fe belongs to the model ferromagnets and the electronic structure of $\mathrm{Fe}$ has been intensively studied by experiments and theory in the last decades [35-43, 45-47], to the best of our knowledge, the only spectroscopic proof of a modification of the electronic band structure as a response to a change of the magnetization direction was provided for $\mathrm{Fe}(110)$ thin films in the scanning tunneling spectroscopy study of Bode et al. [8]. Up to now, $k$-resolved experimental observations of the magnetization-related changes of the electronic band structure of a ferromagnetic film were reported only for surface- or interface-related electronic states such as the surface state of oxidized $\mathrm{Gd}(0001)$ [48] or quantum well states in $\mathrm{Co} / \mathrm{W}(110)$ [49]. In these works, the observed effect was interpreted in relation to the so-called Rashba term $[50,51]$ in the total Hamiltonian of the system. In a theoretical study of the TAMR effect in $\mathrm{Fe}(001)$ [36], the resonant surface bands (not observed in our experiment) were shown to depend on the magnetization direction, which was also attributed to the Rashba effect.

Rashba interaction occurs when an electron moves in a system that lacks structural inversion symmetry (all surfaces and interfaces), in the presence of an electric field and spinorbit interaction [51]. Because in the reference frame of the electron an electric field is seen as an effective magnetic field, a shift of the electron energies occurs. Rashba interaction of the electron with spin and momentum $\hbar \vec{k}$ in the electric field directed along the $z$ axis $\left(\vec{e}_{z}\right)$ is expected, according to $\Delta E=\alpha_{R}\left(\vec{e}_{z} \times \vec{k}\right)$, where $\alpha_{R}$ represents the Rashba parameter, which depends on the strength of the electric field and spin-orbit interaction [51]. In the case of ferromagnetic materials, for the magnetization along $k_{y}$, the Rashba interaction shifts the electronic bands along the $k_{x}$ axis in opposite directions for minority and majority bands
[48]. Therefore, from the symmetry point of view, it resembles the situation discussed for the surface electronic structure of $\mathrm{Fe}(001)$ (Fig. 4) and observed in our experiment.

In principle, the Rashba interaction affects electronic states that are localized near surfaces or interfaces; typical examples are (i) two-dimensional electron gas and (ii) surface states of the high- $Z$ crystalline materials. In our experiment, we observed that the magnetization direction modifies the bulk electronic states of $\mathrm{Fe}(001)$. Because of the surface sensitivity of ARPES (equal to few monolayers), the bulk electronic bands visible in our experiment were probed in the near-surface region. Rashba-type effects for bulk bands were observed before for the occupied bulk bands of $\mathrm{Bi}(111)$ [52] and unoccupied bulk bands of $\mathrm{Au}(111)$ [53], and they were explained as a result of the reflection of the Bloch states from the surface [54]. Therefore, we speculate that the experimentally observed surfacelike symmetry is related to the Rashba-type effect acting on the near-surface part of the bulk electronic states.

\section{SUMMARY AND CONCLUSIONS}

The manipulation of the Fermi surface using an external magnetic field was demonstrated for a prototypical ferromagnet, $\mathrm{Fe}(001)$. We have shown comprehensive ARPES data with clear modifications of the bulk electronic bands of $\mathrm{Fe}(001)$ in response to the remanent change of the magnetization direction.

The experimental electronic band structure of $\mathrm{Fe}(001)$ was found to match the results of the $G W$ first-principles theoretical calculations well for bulk bcc Fe combined with the assumptions of the free-electron final-state model, which allowed the identification of the experimentally observed electronic bands. Results of the one-step model photoemission calculations reproduced symmetries observed in the experiment for different magnetization directions, which indicates that the observed bulk electronic bands are modified by the proximity of the surface. The observed symmetries reveal the interplay between exchange coupling and spin-orbit coupling in the experimental configuration where surface and in-plane remanent magnetization contribute to the symmetry breaking. The observed symmetries agree with the ones expected for the Rashba-type effect. ARPES spectra were found to bear fingerprints of the distinct magnetization direction, which means that a new way of determining the in-plane magnetization direction based on the photoemission spectra has been identified.

We interpret the observed effect as a result of the opening of spin-orbit interaction- and magnetization-related band gaps, the existence of which is essential for the emergence of fundamental magnetic phenomena such as magnetocrystalline anisotropy, anisotropic magnetoresistance, the anomalous Hall effect, and x-ray magnetic linear dichroism. What is more, the detected electronic band gaps might play a substantial role in the spin dynamics. Our finding 
shows that, contrary to common belief, spin-orbit coupling cannot be neglected in the analysis of electronic properties, even for elements as light as $3 d$ ferromagnets.

The results presented here set a new paradigm for the investigations of the spin-orbit effects in the spintronic materials. The same methodology could be used to test different materials and material combinations to judge their potential for TAMR or electric-field magnetization switching, allowing the insightful, bottom-up design of future spintronic devices.

\section{ACKNOWLEDGMENTS}

This work was supported by the Helmholtz Association's Initiative and Networking Fund and the Alexander von Humboldt Foundation. We thank B. Küpper and A. Bremen for technical assistance. H.E., J. M., J. B., and S. B. thank the BMBF (05K13WMA), the DFG (FOR 1346), and the COST Action MP 1306 for financial support. J. M. also thanks Ministerstvo Školství, Mládeže a Tělovýchovy (Project CENTEM (LO1402) and CENTEM PLUS (CZ.1.05/2.100/03.0088)) of Czech Republic for financial assistance.

\section{APPENDIX A: CHEMICAL COMPOSITION OF THE SURFACE}

To address the problem of the possible diffusion of the $\mathrm{Au}$ atoms towards the Fe surface in our system, we performed a supplementary study combining ultraviolet photoemission spectroscopy (UPS) using He I emission line $(h \nu=21.2 \mathrm{eV})$ and $\mathrm{x}$-ray photoelectron spectroscopy (XPS) [Figs. 6(a) and 6(b), respectively]. The 100-ML Fe film, deposited on $\mathrm{Au}(001)$ according to the recipe used in our study, was subsequently annealed, reaching the annealing temperature of $450{ }^{\circ} \mathrm{C}$ after a few annealing steps. After each preparation step, both XPS and UPS spectra were collected.

UPS spectra show characteristic features of the $\mathrm{Au}$ valence band ( $\mathrm{Au} 5 d$ states at $E_{B}=3-4 \mathrm{eV}$ ), which can be easily identified in the spectrum collected for the clean $\mathrm{Au}(001)$ surface [top spectrum in Fig. 6(a)], as well as the strong spectral intensity at the Fermi level, originating from the Fe $3 d$ states [easily recognizable for all the spectra obtained after Fe deposition, Fig. 6(a)]. The XPS spectra were collected in the binding energy region, including $\mathrm{Au} 4 f\left[E_{B}\left(\mathrm{Au} 4 f_{7 / 2}\right)=84.4 \mathrm{eV}\right]$ as well as $\mathrm{Fe} 3 p$ $\left(E_{B}=53.4 \mathrm{eV}\right)$ core-level states. The Fe $3 s$ state $\left(E_{B}=91.2 \mathrm{eV}\right)$ partially overlaps with the Au $4 f$ line. On the freshly deposited Fe film, we see no spectral signatures characteristic of $\mathrm{Au}$, in the UPS or XPS measurements. Mild annealing does not introduce any noticeable changes in this respect (spectrum measured after annealing to $100{ }^{\circ} \mathrm{C}$ ). After annealing to $350{ }^{\circ} \mathrm{C}$, UPS spectra reveal the appearance of the spectral intensity below $E_{B}=3 \mathrm{eV}$, with the accompanying occurrence of (a)

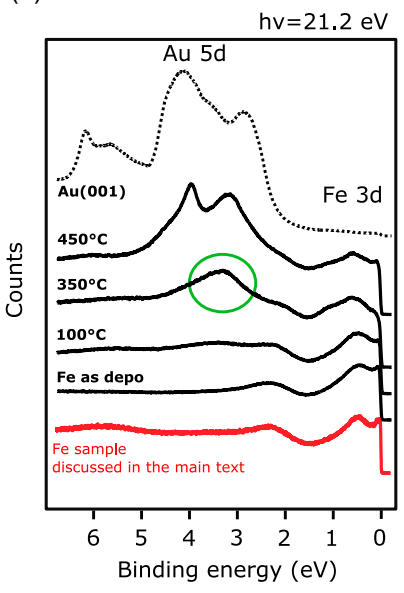

(b)

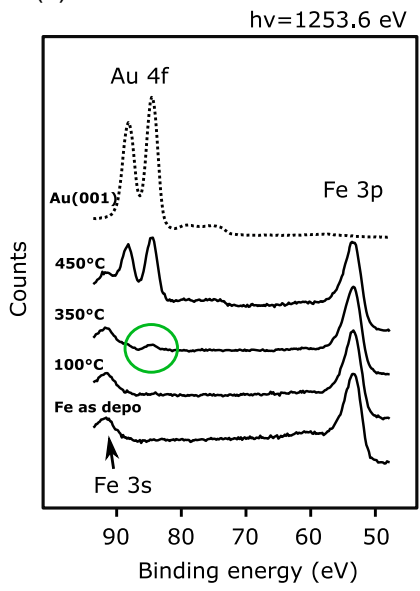

FIG. 6. Results of the chemical characterization of the Fe surface. A 100-ML Fe film was deposited at $50 \mathrm{~K}$ and annealed in steps to the indicated temperatures. (a) UPS spectra measured along normal emission using $\mathrm{He}$ emission line $(h \nu=21.2 \mathrm{eV})$. The red spectrum (bottom of the figure) was collected during the measurements described in the main part of the article. The dotted spectrum at the top represents clean $\mathrm{Au}(001)$ surface. (b) Simultaneously collected XPS spectra.

the Au $4 f$ state in the XPS measurement [both marked with green circles in Fig. 6(a) and 6(b)]. Annealing to higher temperatures $\left(450{ }^{\circ} \mathrm{C}\right)$ results in the increase of the $\mathrm{Au}$ amount detected near the sample surface.

The comparison of the relative intensities of the $\mathrm{Au} 4 f$ and $\mathrm{Fe} 3 p$ lines indicates that the amount of $\mathrm{Au}$ detected after annealing to $350{ }^{\circ} \mathrm{C}$, and $450{ }^{\circ} \mathrm{C}$ corresponds to approximately $0.1 \mathrm{ML}$ and $1 \mathrm{ML}$, respectively. This estimate was made using the $\mathrm{Au} 4 f / \mathrm{Fe} 3 p$ intensity ratio for the bulk signals equal to 4.3 (value calculated according to Ref. [55]) and the inelastic mean-free paths equal to $13.80 \AA$ for kinetic energy of the Au $4 f$ core-level electrons in $\mathrm{Au}, 18.80 \AA$ for kinetic energy of the $\mathrm{Fe} 3 p$ electrons in $\mathrm{Fe}$, and $14.06 \AA$ for the kinetic energy of $\mathrm{Fe} 3 p$ electrons in $\mathrm{Au}$ (found using the TPP2M formula [56,57]).

As the UPS spectra collected during experiments and discussed in the main text do not contain any signs of the $\mathrm{Au} 5 d$ states [red line at the bottom of Fig. 6(a)], we conclude that the Fe surface was free from the Au adatoms, within the sensitivity limit of our spectroscopic determination, which we can safely assume to be of the order of $0.1 \mathrm{ML}$ Au.

\section{APPENDIX B: DETAILS OF THE FREE-ELECTRON FINAL-STATE MODEL}

According to the free-electron final-state model, excitation with a fixed photon energy leads to photoemission from well-defined regions of the BBZ. Following the formula that relates the perpendicular component of the electron wave vector $\left(k_{\perp}\right)$ with the kinetic energy of 
the photoemitted electrons $\left(E_{\text {kin }}\right)$, the emission angle $(\Theta)$, and the so-called inner potential $\mathrm{V}_{0}$ (adjusted to be equal to $7 \mathrm{eV}), k_{\perp}=\sqrt{\left(2 m / \hbar^{2} E_{\mathrm{kin}} \cos ^{2}(\Theta)+V_{0}\right)}$, we estimate that the result of the experiment performed using $h \nu=$ $16.8 \mathrm{eV}$ corresponds to a cut of the BBZ zone at $k_{\perp} \sim$ $2.2 \AA^{-1}$ when $\Theta=0^{\circ}$. The calculated $G W$ dispersions and the derived cuts through the Fermi surface [Figs. 3(a), 3(c), 3(f), and 5(e)] are shown for constant $k_{\perp} \sim 2.2 \AA^{-1}$, i.e., not including the $k_{\perp}(\Theta)$ dependence. We found that such derived bands and constant energy cuts result in the best agreement with the experimentally observed dispersions.

\section{APPENDIX C: DETAILS OF ONE-STEP MODEL CALCULATIONS}

For the calculation of the ground-state properties, we used a fully relativistic multiple scattering method in the framework of density functional theory (KKR) [26]. We used the tight-binding (TB) approximation for an effective calculation of the surface properties. This method provides a fast convergence of the calculation of the so-called TB structure constants [58]. The calculations of the selfconsistent potentials have been carried out in the atomic sphere approximation. For the exchange-correlation functional, we used the parametrization of Vosko, Wilk, and Nusair [59].

Using this method, we were able to investigate the influence of the surface contribution to the resulting ARPES spectra. This is done by tuning the multiple scattering between surface barrier and 2D semi-infinite system as follows. The bulk potentials are well ordered according to the two-dimensional crystal structure of the given surface. This means that the spin-orbit interaction determined from the single ion core potentials, which contain no information about $k_{\perp}$, is transformed into a $k_{\|}$splitting of the surface state through multiple scattering. This scattering procedure is realized by the bulk reflection matrix $B$, which is developed into the two-dimensional reciprocal lattice vectors of the corresponding surface. This matrix represents the complete electronic structure information of the semi-infinite bulk. The scattering is, in first order, proportional to $B$. When the off-diagonal elements of $B$ are reduced, the transfer of spin-orbit interaction from the bulk to the surface layer is diminished. For more details, the reader is referred to Ref. [60].

\section{APPENDIX D: DETAILS OF $G W$ CALCULATIONS}

For the starting point of $G W$, we used the LDA exchangecorrelation functional [61] and the code FLEUR [25]. For this, we used an angular-momentum cutoff of $l_{\max }=8$ in the atomic spheres and a plane-wave cutoff of $5.0 \mathrm{bohr}^{-1}$ in the interstitial region. We used the experimental lattice parameter $2.87 \AA$ from Ref. [62], and the semicore $3 s$ and
$3 p$ states of Fe were treated as valence orbitals by the use of local orbitals. The mixed product basis $[23,63]$ used in the $G W$ calculation was constructed with an angular momentum cutoff of $l_{\max }=4$ and a plane-wave cutoff of $3.0 \mathrm{bohr}^{-1}$. We used 170 unoccupied bands and a $10 \times 10 \times 10 \quad k$-point sampling of the Brillouin zone. This leads to energy differences and a Fermi energy converged up to $10 \mathrm{meV}$. Two additional local orbitals per angular momentum up to $l=3$ were included to describe high-lying states accurately and to avoid linearization errors $[64,65]$.

[1] Z. Fang, N. Nagaosa, K. S. Takahashi, A. Asamitsu, R. Mathieu, T. Ogasawara, H. Yamada, M. Kawasaki, Y. Tokura, and K. Terakura, The Anomalous Hall Effect and Magnetic Monopoles in Momentum Space, Science 302, 92 (2003).

[2] Y. Yao, L. Kleinman, A. H. MacDonald, J. Sinova, T. Jungwirth, D. Wang, E. Wang, and Q. Niu, First Principles Calculation of Anomalous Hall Conductivity in Ferromagnetic bcc Fe, Phys. Rev. Lett. 92, 037204 (2004).

[3] L. Berger, Influence of Spin-Orbit Interaction on the Transport Processes in Ferromagnetic Nickel Alloys, in the Presence of a Degeneracy of the 3D Band, Physica (Amsterdam) 30, 1141 (1964).

[4] G. van der Laan, Microscopic Origin of Magnetocrystalline Anisotropy in Transition Metal Thin Films, J. Phys. Condens. Matter 10, 3239 (1998).

[5] M. Weisheit, S. Fähler, A. Marty, Y. Souche, C. Poinsignon, and D. Givord, Electric Field-Induced Modifications of Magnetism in Thin-Film Ferromagnets, Science 315, 349 (2007).

[6] T. Maruyama et al., Large Voltage-Induced Magnetic Anisotropy Change in a Few Atomic Layers of Iron, Nat. Nanotechnol. 4, 158 (2009).

[7] K. Nakamura, R. Shimabukuro, Y. Fujiwara, T. Akiyama, T. Ito, and A. J. Freeman, Giant Modification of the Magnetocrystalline Anisotropy in Transition-Metal Monolayers by an External Electric Field, Phys. Rev. Lett. 102, 187201 (2009).

[8] M. Bode, S. Heinze, A. Kubetzka, O. Pietzsch, X. Nie, G. Bilhmayer, S. Blügel, and R. Wiesendanger, MagnetizationDirection-Dependent Local Electronic Structure Probed by Scanning Tunneling Spectroscopy, Phys. Rev. Lett. 89, 237205 (2002).

[9] C. Gould, C. Rüster, T. Jungwirth, E. Girgis, G. M. Schott, R. Giraud, K. Brunner, G. Schmidt, and L. W. Molenkamp, Tunneling Anisotropic Magnetoresistance: A Spin-ValveLike Tunnel Magnetoresistance Using a Single Magnetic Layer, Phys. Rev. Lett. 93, 117203 (2004).

[10] J. Moser, A. Matos-Abiague, D. Schuh, W. Wegscheider, J. Fabian, and D. Weiss, Tunneling Anisotropic Magnetoresistance and Spin-Orbit Coupling in Fe/GaAs/Au Tunnel Junctions, Phys. Rev. Lett. 99, 056601 (2007).

[11] B. G. Park et al., A Spin-Valve-Like Magnetoresistance of an Antiferromagnet-Based Tunnel Junction, Nat. Mater. 10, 347 (2011). 
[12] J. Velev, R. F. Sabirianow, S. S. Jaswal, and E. Y. Tsymbal, Ballistic Anisotropic Magnetoresistance, Phys. Rev. Lett. 94, 127203 (2005).

[13] G. van der Laan, Magnetic Linear X-Ray Dichroism as a Probe of the Magnetocrystalline Anisotropy, Phys. Rev. Lett. 82, 640 (1999).

[14] M. Pickel, A. B. Schmidt, F. Giesen, J. Braun, J. Minár, H. Ebert, M. Donath, and M. Weinelt, Spin-Orbit Hybridization Points in the Face-Centered-Cubic Cobalt Band Structure, Phys. Rev. Lett. 101, 066402 (2008).

[15] D. Steiauf and M. Fähnle, Elliott-Yafet Mechanism and the Discussion of Femtosecond Magnetization Dynamics, Phys. Rev. B 79, 140401 (2009).

[16] J. Fabian and S. Das Sarma, Spin Relaxation of Conduction Electrons in Polyvalent Metals: Theory and a Realistic Calculation, Phys. Rev. Lett. 81, 5624 (1998).

[17] B. Zimmermann, P. Mavropoulos, S. Heers, N. H. Long, S. Blügel, and Y. Mokrousov, Anisotropy of Spin Relaxation in Metals, Phys. Rev. Lett. 109, 236603 (2012).

[18] S. D. Bader and E. R. Moog, Magnetic Properties of Novel Epitaxial Films, J. Appl. Phys. 61, 3729 (1987).

[19] F. J. Himpsel, Fe on Au (100): Quantum-Well States Down to a Monolayer, Phys. Rev. B 44, 5966 (1991).

[20] D. Wilgocka-Ślęzak, K. Freindl, A. Kozioł, K. Matlak, M. Rams, N. Spiridis, M. Ślęzak, T. Ślęzak, M. Zając, and J. Korecki, Thickness-Driven Polar Spin Reorientation Transition in Ultrathin Fe/Au (001) Films, Phys. Rev. B 81, 064421 (2010).

[21] R. Hammer, A. Sander, S. Förster, M. Kiel, K. Meinel, and W. Widdra, Surface Reconstruction of Au (001): HighResolution Real-Space and Reciprocal-Space Inspection, Phys. Rev. B 90, 035446 (2014).

[22] L. Plucinski, A. Oelsner, F. Matthes, and C. M. Schneider, A Hemispherical Photoelectron Spectrometer with 2-Dimensional Delay-Line Detector and Integrated SpinPolarization Analysis, J. Electron Spectrosc. Relat. Phenom. 181, 215 (2010).

[23] C. Friedrich, S. Blügel, and A. Schindlmayr, Efficient Implementation of the GW Approximation within the AllElectron FLAPW Method, Phys. Rev. B 81, 125102 (2010).

[24] J. P. Perdew, K. Burke, and M. Ernzerhof, Generalized Gradient Approximation Made Simple, Phys. Rev. Lett. 77, 3865 (1996).

[25] See http://www.flapw.de.

[26] H. Ebert et al., The Munich SPR-KKR Package, Version 6.3, http://olymp.cup.uni-muenchen.de/ak/ebert/SPRKKR, 2012.

[27] J. B. Pendry, Low Energy Electron Diffraction (Academic Press, London, 1974).

[28] J. B. Pendry, Theory of Photoemission, Surf. Sci. 57, 679 (1976).

[29] J. F. L. Hopkinson, J. B. Pendry, and D. J. Titterington, Calculation of Photoemission Spectra for Surfaces of Solids, Comput. Phys. Commun. 19, 69 (1980).

[30] J. Braun, The Theory of Angle-Resolved Ultraviolet Photoemission and Its Applications to Ordered Materials, Rep. Prog. Phys. 59, 1267 (1996).

[31] J. Braun, New Developments in UPS and XPS from Ferromagnetic Materials, in Band-Ferromagnetism: Ground-State and Finite-Temperature Phenomena, edited by K. Baberschke, M. Donath, and W. Nolting (Springer, Berlin, 2001).

[32] G. Malmström and J. Rundgren, A Program for Calculation of the Reflection and Transmission of Electrons through a Surface Potential Barrier, Comput. Phys. Commun. 19, 263 (1980).

[33] W. H. Butler, X.-G. Zhang, T. C. Schulthess, and J. M. MacLaren, Spin-Dependent Tunneling Conductance of $\mathrm{Fe} / \mathrm{MgO} / \mathrm{Fe}$ Sandwiches, Phys. Rev. B 63, 054416 (2001).

[34] M. I. Katsnelson and A. I. Lichtenstein, LDA $++A p$ proach to the Electronic Structure of Magnets: Correlation Effects in Iron, J. Phys. Condens. Matter 11, 1037 (1999).

[35] Joseph A. Stroscio, D. T. Pierce, A. Davies, R. J. Celotta, and M. Weinert, Tunneling Spectroscopy of bcc (001) Surface States, Phys. Rev. Lett. 75, 2960 (1995).

[36] A. N. Chantis, K. D. Belashchenko, E. Y. Tsymbal, and M. van Schilfgaarde, Tunneling Anisotropic Magnetoresistance Driven by Resonant Surface States: First-Principles Calculations on an Fe (001) Surface, Phys. Rev. Lett. 98, 046601 (2007).

[37] L. Plucinski, Y. Zhao, C. M. Schneider, B. Sinkovic, and E. Vescovo, Surface Electronic Structure of Ferromagnetic Fe (001), Phys. Rev. B 80, 184430 (2009).

[38] A. M. Turner and J. L. Erskine, Surface Electronic Properties of Fe (100), Phys. Rev. B 30, 6675 (1984).

[39] A. M. Turner and J. L. Erskine, Magnetic Exchange Splitting and Band Dispersion of Surface States on Fe (100), Phys. Rev. B 28, 5628 (1983).

[40] E. Vescovo, O. Rader, and C. Carbone, Spin-Polarized Surface States of Fe (100), Phys. Rev. B 47, 13051 (1993).

[41] Ch. Eibl, A. B. Schmidt, and M. Donath, Appearance of the Minority $d z^{2}$ Surface State and Disappearance of the Image-Potential State: Criteria for Clean Fe(001), Phys. Rev. B 86, 161414 (2012).

[42] S. De Rossi, L. Duo, and F. Cicacci, Polarized Unoccupied States of Oxygen on $\mathrm{Fe}(001)$, Europhys. Lett. 32, 687 (1995).

[43] L.-N. Tong, F. Matthes, M. Müller, C. M. Schneider, and Ch. G. Lee, Influence of $\mathrm{MgO}$ Overlayers on the Electronic States of Fe(001) Thin Films Grown on GaAs (001), Phys. Rev. B 77, 064421 (2008).

[44] edited by, Th. Hahn, International Tables for Crystallography, Volume A: Space-group symmetry (Wiley, New York, 2006).

[45] M. Sawada, A. Kimura, and A. Kakizaki, Spin-Dependent Occupied Surface State of Fe (001), Solid State Commun. 109, 129 (1998).

[46] B. Ackermann, R. Feder, and E. Tamura, Fully Relativistic Band Structure of Ferromagnetic Fe and Gd, J. Phys. F 14, L173 (1984).

[47] P. Heimann and H. Neddermeyer, Photoemission Study of Single-Crystal Faces of Iron, Phys. Rev. B 18, 3537 (1978).

[48] O. Krupin, G. Bihlmayer, K. Starke, S. Gorovikov, J. E. Prieto, K. Döbrich, S. Blügel, and G. Kaindl, Rashba Effect at Magnetic Metal Surfaces, Phys. Rev. B 71, 201403 (2005).

[49] P. Moras, G. Bihlmayer, P. M. Sheverdyaeva, S. K. Mahatha, M. Papagno, J. Sánchez-Barriga, O. Rader, L. Novinec, S. Gardonio, and C. Carbone, Magnetization-Dependent Rashba Splitting of Quantum Well States at the Co/W Interface, Phys. Rev. B 91, 195410 (2015). 
[50] Y. A. Bychkov and E. I. Rashba, Properties of a 2D Electron Gas with Lifted Spectral Degeneracy, JETP Lett. 39, 78 (1984).

[51] G. Bihlmayer, Y. M. Koroteev, P. M. Echenique, E. V. Chulkov, and S. Blügel, The Rashba-Effect at Metallic Surfaces, Surf. Sci. 600, 3888 (2006).

[52] A. Kimura, E. E. Krasovskii, R. Nishimura, K. Miyamoto, T. Kadono, K. Kanomaru, E. V. Chulkov, G. Bihlmayer, K. Shimada, H. Namatame, and M. Taniguchi, Strong RashbaType Spin Polarization of the Photocurrent from Bulk Continuum States: Experiment and Theory for Bi (111), Phys. Rev. Lett. 105, 076804 (2010).

[53] S. N. P. Wissing, C. Eibl, A. Zumbülte, A. B. Schmidt, J. Braun, J. Minár, H. Ebert, and M Donath, Rashba-Type Spin Splitting at Au (111) Beyond the Fermi Level: The Other Part of the Story, New J. Phys. 15, 105001 (2013).

[54] E. E. Krasovskii and E. V. Chulkov, Rashba Polarization of Bulk Continuum States, Phys. Rev. B 83, 155401 (2011).

[55] C. D. Wagner, L. E. Davis, M. V. Zeller, J. A. Taylor, R. H. Raymond, and L. H. Gale, Empirical Atomic Sensitivity Factors for Quantitative Analysis by Electron Spectroscopy for Chemical Analysis, Surf. Interface Anal. 3, 211 (1981).

[56] S. Tanuma, C. J. Powell, and D. R. Penn, Calculations of Electron Inelastic Mean Free Paths, Surf. Interface Anal. 21, 165 (1994).

[57] S. Tougaard, QUASES-IMFP-TPP2M Version 3.0, QuasesTougaard Inc., 2000-2010.
[58] R. Zeller, P. H. Dederichs, B. Újfalussy, L. Szunyogh, and P. Weinberger, Theory and Convergence Properties of the Screened Korringa-Kohn-Rostoker Method, Phys. Rev. B 52, 8807 (1995).

[59] S. H. Vosko, L. Wilk, and M. Nusair, Accurate SpinDependent Electron Liquid Correlation Energies for Local Spin Density Calculations: A Critical Analysis, Can. J. Phys. 58, 1200 (1980).

[60] A. Nuber, J. Braun, F. Forster, J. Minár, F. Reinert, and H. Ebert, Surface Versus Bulk Contributions to the Rashba Splitting in Surface Systems, Phys. Rev. B 83, 165401 (2011).

[61] J. P. Perdew and A. Zunger, Self-Interaction Correction to Density-Functional Approximations for Many-Electron Systems, Phys. Rev. B 23, 5048 (1981).

[62] R. W. G. Wyckoff, Crystal Structures (J. Wiley and Sons, New York, 1963).

[63] T. Kotani and M. van Schilfgaarde, All-Electron GW Approximation with the Mixed Basis Expansion Based on the Full-Potential LMTO Method, Solid State Commun. 121, 461 (2002).

[64] C. Friedrich, A. Schindlmayr, S. Blügel, and T. Kotani, Elimination of the Linearization Error in $G W$ Calculations Based on the Linearized Augmented-Plane-Wave Method, Phys. Rev. B 74, 045104 (2006).

[65] C. Friedrich, M. C. Müller, and S. Blügel, Band Convergence and Linearization Error Correction of All-Electron GW Calculations: The Extreme Case of Zinc Oxide, Phys. Rev. B 83, 081101 (2011). 